\title{
1 Comparative Study of Deep Generative Models on 2 Chemical Space Coverage
}

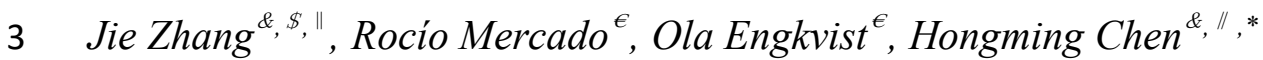

$4 \quad{ }^{\&}$ Guangdong Provincial Key Laboratory of Laboratory Animals, Guangdong Laboratory

5 Animals Monitoring Institute, Guangzhou, 510663, P. R. China

$6 \quad{ }^{\$}$ State Key Laboratory of Respiratory Disease, Guangzhou Institutes of Biomedicine and Health,

7 Chinese Academy of Sciences, Guangzhou 510530, P. R. China

8 "Bioland Laboratory (Guangzhou Regenerative Medicine and Health - Guangdong

9 Laboratory), Guangzhou 510530, P. R. China

$10{ }^{\epsilon}$ Hit Discovery, Discovery Sciences, R\&D, AstraZeneca, Gothenburg 43183, Sweden

$11 \quad{ }^{*}$ Correspondence e-mail: chen_hongming@grmh-gdl.cn

12 Abstract

13 In recent years, deep molecular generative models have emerged as novel methods for de novo 14 molecular design. Thanks to the rapid advance of deep learning techniques, deep learning 15 architectures such as recurrent neural networks, variational autoencoders, and adversarial networks, 16 have been employed for constructing generative models. However, so far the metrics used to 17 evaluate these deep generative models are not discriminative enough to separate the performance 18 of various state-of-the-art generative models. This work presents a novel metric for evaluating 19 deep molecular generative models; this new metric is based on the chemical space coverage of a 
reference database, and compares not only the molecular structures, but also the ring systems and functional groups, reproduced from a reference dataset of a $1 \mathrm{M}$ subset of GDB-13. The performance of 7 different molecular generative models was compared by calculating their structure and substructure coverage of the GDB-13 database while using the 1M subset for training. The result shows that the performance of various generative models varies significantly using the benchmarking metrics introduced herein, such that generalization capability of the generative model can be clearly differentiated. Additionally, the coverage of ring systems and functional groups existing in GDB-13 was also compared between the models. Our study provides a useful new metric that can be used for evaluating and comparing generative models.

\section{Introduction}

Deep learning has been successfully used in many fields to learn relationships that are too complex

to learn using traditional computer algorithms, including early image classification,,$^{1,2}$ facial recognition, and music recognition. ${ }^{3}$ Deep learning even surpasses the performance of human experts in some challenging tasks, such as playing GO. ${ }^{4}$ Moreover, deep generative models play important roles in tasks like music composition, ${ }^{5}$ image generation, ${ }^{6}$ and language translation. ${ }^{7}$ In the last five years, deep generative modeling has also been applied in the fields of cheminformatics and molecular design. One interesting example is using deep neural networks for compound structure generation..$^{8 \cdot 11}$

The number of chemically feasible, drug-like molecules has been estimated to be on the order of $10^{60}-10^{100}$ compounds. ${ }^{12}$ For such a large chemical space, it is clearly impossible to synthesize and test every compound for pharmaceutical applications. To efficiently explore the space, molecular 
41 generative models have emerged in recent years with the aim of better navigating through this 42 huge chemical space for de novo molecule design.

43 De novo molecular design has long been put forward as a way to accelerate the drug discovery 44 process as it is expected to save time and resources in drug discovery, where it can take over a 45 decade and billions of dollars in investment to bring a single drug to market. ${ }^{13}$ Historically, de novo 46 design methods have been mainly rule-driven and used brute force algorithms to achieve their

47 goal. ${ }^{14}$ For example, creating a virtual library using fixed rules and building blocks, then scoring 48 each compound in the virtual library to find the best compound. Genetic algorithm based 49 algorithms were also proposed to tackle the de novo design issue..$^{15,16}$ In contrast, deep generative 50 molecular design is the concept of generating molecules using deep neural networks. Deep 51 generative models are data-driven methods which generate compound structures by learning the 52 underlying probability distributions in a compound dataset instead of screening existing databases 53 for molecules that fit the desired profile. Deep generative models are powerful as they allow 54 chemists to bypass models using hard-coded chemical rules which do not scale to larger datasets. 55 Furthermore, not all chemical rules are easy to define. Using deep generative models, one can 56 avoid enumerating all possible structures for a given application and then screening them (a 57 daunting task). Instead, one can simply train a model using known compounds, and sample the 58 model for the desired set of properties (e.g. ADMET profile) to get out promising structures. De 59 novo generative models can generate structures that are in significantly narrower, but more 60 promising, regions of chemical space. Moreover, deep learning methods can take advantage of all 61 the information available in ever-increasing large public datasets, thanks to automation 62 technologies used in high-throughput screening and parallel synthesis. ${ }^{17}$ 
In recent years, many molecular generative models have been published, such as CharRNN, VAE, and REINVENT, which are remarkable at sampling molecules both in- and outside the training set used to learn chemistry rules..11,1821 It is worth noting that CharRNN was introduced as a general language model at the first place. However, similar architectures are also successfully applied in molecular generative models, e.g. REINVENT adopted a similar architecture with reinforcement learning. ${ }^{2226} \mathrm{VAE}$ is a general architecture that has a wide range of applications in many generative models and tasks. ${ }^{2-29}$ In current study, we adopts the implementation of CharRNN and VAE provided by the MOSES. ${ }^{10}$ Notably, many of these generative models have been benchmarked using existing "distribution-based" metrics implemented in open-source programs such as MOSES $^{10}$ or GuacaMol ${ }^{30}$ However, these metrics are in general non-discriminative as many of these state-of-the-art (SOTA) models perform quite well across all the included metrics, such that it is difficult to compare them and gain a deep understanding of each model's strengths and weaknesses. We previously proposed a new metric: the percent coverage of functional groups present in GDB-13. ${ }^{31}$ As an extension of our previous work, ${ }^{32}$ we apply the idea as a way for benchmarking the performance of multiple generative models. GDB-13 contains in total $975,820,210$ structures, which enumerate small organic molecules containing up to 13 atoms of C, $\mathrm{N}, \mathrm{O}, \mathrm{S}$, and $\mathrm{Cl}$ by following simple chemical stability and synthetic feasibility rules. ${ }^{32}$ The generalization capability of deep generative models is assessed by computing how much of the whole GDB-13 can be recovered by a model trained from a small GDB-13 subset.

Substructure has long been used to characterize the composition of compounds. One concept is the so-called functional group, frequently used in many fields in chemistry, including medicinal chemistry. A functional group is defined as a subset of connected atoms in a molecule that in some way endows specific intrinsic properties (or functions) to a molecule. Furthermore, the presence 
or absence of a functional group in a molecule could determine whether a molecule will react in a given reaction. Some of the most common groups in medicinal chemistry include amides $(\mathrm{RC}(=\mathrm{O}) \mathrm{NR}$ 'R"), ethers (R-O-R'), and amines (RR'NR"), where $\mathrm{R}, \mathrm{R}$, and $\mathrm{R}$ " represent organic groups or hydrogen atoms. ${ }^{33}$ Another substructure-based concept is the ring system; ring systems are the key components of molecular scaffolds. They play an important role in a molecule's observed properties, such as the electronics, scaffold rigidity, molecular reactivity, and toxicity. On average six new ring systems enter the drug space each year and approximately $28 \%$ of new drugs contain a new ring system. ${ }^{34}$ We investigated the percentage of chemical space covered in terms of full structures, functional groups, and ring systems by published SOTA generative models. The size of GDB-13 was hypothesized to be large enough to highlight differences between the various models.

Four major classes of deep generative models are benchmarked and studied in this work, including those based on recurrent neural networks (RNNs), autoencoder (AE) based networks, generative adversarial networks (GANs), and graph neural networks (GNNs). The deep generative models based on RNNs include REINVENT ${ }^{18,32,35}$ and CharRNN, ${ }^{25}$ which use SMILES as the input and output strings. VAE $,^{36} \mathrm{AAE},{ }^{21,37} \mathrm{ORGAN},{ }^{20}$ and LatentGAN" adopt either an $\mathrm{AE}$ or GAN for structure generation using SMILES. Besides the SMILES-based generative models, one graph-based generative model, GraphINVENT ${ }^{38}$ which uses GNNs in its core architecture, is also included in the benchmark study. An effort was made to cover most of the major types of generative model architectures in this study, in the hope that this would provide a comprehensive comparison for existing generative models.

\section{Methods}


108 Extraction of functional groups and ring systems. To identify functional groups (FG) in the 109 various sets of molecules in this work (generated molecule sets, and GDB-13), the RDKit 110 functional group identification package,,$^{39}$ which is based on an algorithm introduced by Peter Ertl 111 for automatically identifying functional groups, was used ${ }^{40}$ The advantage of the method is that it 112 is not based on manually curated lists of functional groups, and thus can be applied to any chemical 113 series. It is important to note that different chemists have slightly different definitions of what is a 114 functional group; however, as the benchmark introduced here calculated ratios of functional groups 115 in the generated and reference sets, a difference in opinions between chemists shouldn't be relevant. 116 The extraction of compound ring system (RS) was done using RDKit. First, all monocyclic rings 117 were retrieved; monocyclic rings were then fused depending on if individual ring systems shared 118 atoms or not.

119 Generative models. The models studied in this study include CharRNN, REINVENT, AAE, VAE, 120 ORGAN, LatentGAN, and GraphINVENT. The REINVENT code available at the 121 github.com/undeadpixel/reinvent-randomized repo ${ }^{35,41}$ was used; the CharRNN, AAE, VAE, and 122 ORGAN codes available at the MOSES GitHub repository ${ }^{10,42}$ were used; the LatentGAN code 123 available at the github.com/Dierme/latent-gan repository ${ }^{11,43}$ was used. Unlike original implement 124 of LatentGAN, we retrained the embedded Deep Drug Coder (DDC) model with randomly 125 selected 3M molecules from GDB-13 as the encoder and decoder component of LatentGAN. The 126 DDC code available at the github.com/pcko1/Deep-Drug-Coder repository ${ }^{44}$ was used; finally, the 127 GraphINVENT code available at the github.com/MolecularAI/GraphINVENT repository ${ }^{38,45,46}$ was 128 used. All methods except for GraphINVENT are string-based generative models, whereas 129 GraphINVENT is a graph-based generative model. 
Training. The GDB-13 database is used as the reference chemical space for this study. ${ }^{31}$ A one million (1M) molecule subset of GDB-13 was randomly selected and used as the training set for all the generative models. Another $200 \mathrm{~K}$ molecules of GDB-13 were selected as the validation set for calculating the validation loss. During training, a check point model was saved at every epoch. The check point model with the lowest validation loss was chosen as the final model for sampling 1 billion (1B) SMILES.

Hyperparameters. For REINVENT, hyperparameters were taken from the GitHub repo." For CharRNN, AAE, VAE, and ORGAN, the parameters were taken from the models' config file in MOSES GitHub repo without further optimization. For LatentGAN, the default values of parameters in the GitHub repo were adopted."1, ${ }^{43}$ For GraphINVENT, parameters and hyperparameters for the best performing model (cGGNN) in the original publication were used and not further optimized. ${ }^{46}$ Detailed hyperparameters for each model can be found in SI Table S2.

Sampling. Once each model was trained, 1B compounds were sampled from each trained model. The functional groups and ring systems were then identified for all sample sets as well as the full GDB-13 set. All compounds in the analysis were standardized by converting to RDKit canonical SMILES. Molecular graphs generated using GraphINVENT were further sanitized via canonicalizing and aromatizing during the conversion to canonical RDKit SMILES for a more fair comparison to the other models.

Technical details. For models in the MOSES repository and REINVENT, the training was done using Python 3.6 and PyTorch 1.4.4. To accelerate sampling for 1B SMILES, the largest batch size allowed by the GPU memory was adopted; for example, ORGAN, AAE, and VAE adopted a sampling batch size of 25, 000, and CharRNN adopted a sampling batch size of 20,000. Also, LatentGAN was trained using tensorflow-gpu 2.2, which adopted a sampling batch size of 50,000. 
153 All the computations were performed on Linux workstations with GeForce RTX 2080Ti graphic 154 cards using CUDA 10.1. Canonical SMILES and dataset analysis were carried out using RDKit. ${ }^{39}$ 155 The Wasserstein distances ${ }^{49}$ between distributions in Figure 2 were calculated with an in-house 156 script using SciPy. ${ }^{50}$ Finally, GraphINVENT runs using Python 3.6 and PyTorch 1.2.

\section{Results and Discussions}

\section{Analysis of the GDB-13 database}

159 GDB-13 contains theoretically drug-like compounds whose heavy atom count is less than or equal functional groups. The distribution of the occurrence frequency of these ring systems and of the ring systems ( 93\%) and functional groups ( 91\%) appear in GDB-13 less than 20 times.

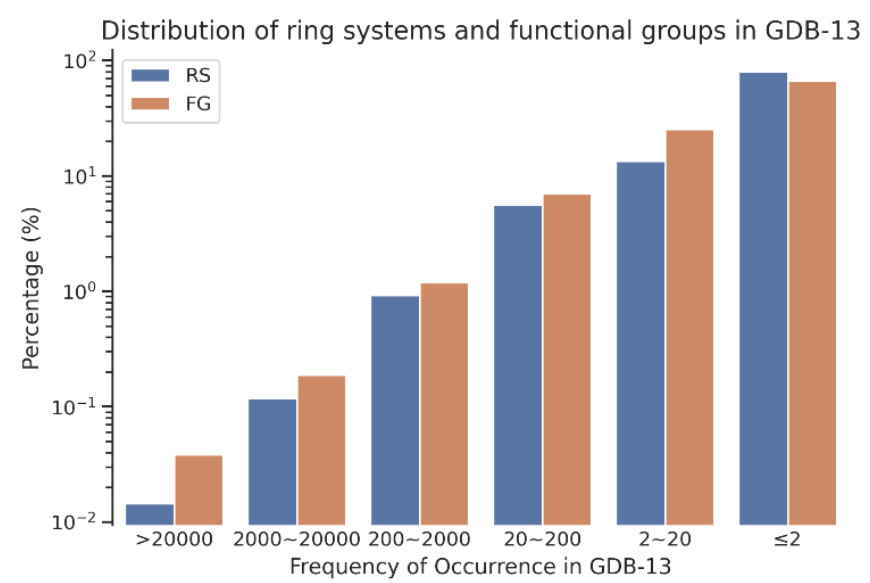

Figure 1. Distribution of ring systems (RS) and functional groups (FG) in GDB-13 according to the frequency of occurrence. Y-axis is the percentage plotted on a logarithmic scale. 


\section{Analysis of the 1M training dataset}

170 One million SMILES were randomly selected from the GDB-13 database for the training set, 171 which corresponds to roughly $0.1 \%$ of the total GDB-13 dataset. The training set contains around $1720.9 \%$ of the ring systems and functional groups in the whole GDB-13 database (Table 1). The 173 coverage of the ring systems and functional groups is nine times as high as the coverage of 174 compounds, which is obviously due to the fact that some ring systems and functional groups occur 175 far more than once in GDB-13, as shown in Figure 1.

176 Table 1. Summary of GDB-13 coverage in the training set, consisting of $1 \mathrm{M}$ randomly selected 177 molecules.

\begin{tabular}{lll}
\hline Item & Counts in the training dataset $(1 \mathrm{M})$ & Coverage of GDB-13 \\
\hline Compounds & $1,000,000$ & $\sim 0.1 \%$ \\
Ring systems & 202,848 & $\sim 0.9 \%$ \\
Functional groups & 38,209 & $\sim 0.9 \%$ \\
\hline
\end{tabular}

(a) Ring Systems (RS)

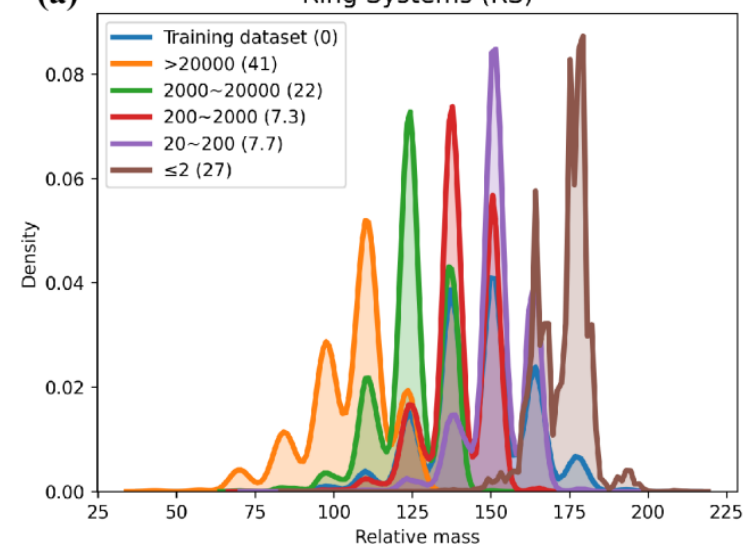

(b)

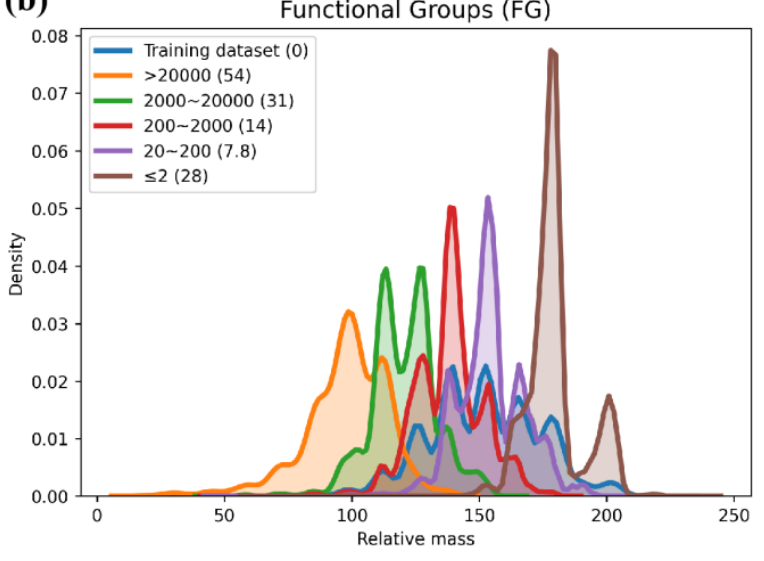

Figure 2. The frament weight distributions for the different substructures in GDB-13. The different

181 colors indicate distributions involving substructures that occur in GDB-13 a similar number of times (i.e. orange is substructures that occur $>20,000$ times in GDB-13, brown is substructures that 
183

184 185 186 187 188 189 190 191

occur $\leq 2$ times). In the key, the numbers in parentheses indicate the Wasserstein distance between the training set distribution and the indicated distribution. (a) Ring systems (RS). (b) Functional groups (FG).

The fragment weights (FWs) of ring systems and functional groups in the training set, grouped by frequency of occurrence, are shown in Figure 2. The FWs here were calculated from the composition of specific ring systems and functional groups rather than the full compound. It is observed that their probability of occurrence decreases with increasing FW. For example, the mean FW of RS and FG which occur with a frequency $>20,000$ is around 100; however, for RS and FG which occur $<=2$ times in GDB-13, the mean FW is around 170. More basic RS and FG, such as $\mathrm{C} 1 \mathrm{CC} 1$ (cyclopropyl) and $\mathrm{C}=\mathrm{O}$ (carbonyl), respectively, tend to have smaller FW compared to complex RS and FG. Furthermore, many complex RS and FG can be built from the basic components via enumeration and combination following the chemical rules extracted from the training dataset.

\section{Training and sampling speed}

All deep molecular generative models were trained with the same training set of $1 \mathrm{M}$ compounds. Each epoch of training took 17-20 min for most models (Figure 3), except CharRNN (28 min) and GraphINVENT (36 min). In general, the training speed of all the models is acceptable. We observed that training SMILES-based models is faster than the graph-based model; this is understandable because the action space of the graph-based model is much larger than any of the SMILES-based models.

Nonetheless, the sampling speed of the generative models was observed to vary significantly. The sampling speed of REINVENT, AAE, ORGAN, and VAE were all above 7000 compounds per second, while the sampling speed of CharRNN, LatentGAN, and GraphINVENT were only 200, 
207 similar architecture of character-level recurrent neural networks. The difference of their 208 performance is mainly due to CharRNN implementation provided by MOSES adopts a larger size 209 of architecture. The detailed hyper parameters are given as Table S2 in the supporting materials.

210 It should also be noted that both training and sampling speeds are strongly related to the batch size that is limited by the memory of the GPU. In current work, the default batch size as specified in

212 the code was used during the training, while for sampling, the largest batch size allowed by the 213 GPU memory was chosen.

214 Given the relatively small size of the training set (1M molecules), all the deep generative models 215 had a tractable training speed. In terms of sampling, the sampling speed was limited by each 216 model's architecture and size; using a larger sampling batch size allowed by a greater GPU 217 memory could boost the sampling speed.
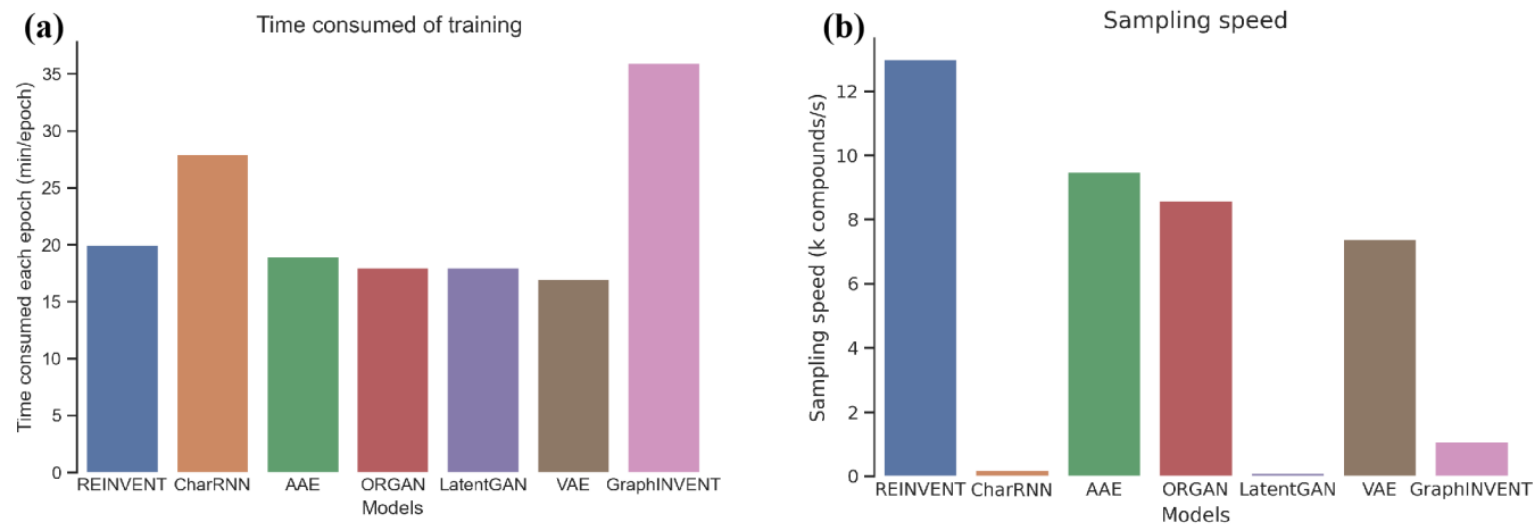

Figure 3. Training and sampling speeds of the generative models benchmarked in this work. (a)

220 Time consumed per epoch during training. (b) Sampling speed, which is the number of 221 SMILES/graphs generated per second (including invalid ones). 
223 We first check the validity of the molecules generated by all the deep generative models, which is 224 defined as the percentage of chemically valid SMILES/graphs in the 1B generated set. Table 2 225 shows that the validity in general is satisfactory for all models, where most models achieve a 226 validity higher than 90 percent. RNN based models (REINVENT and CharRNN) have the highest 227 validity which is above $99.3 \%$ (Table 2). The validity of LatentGAN and GraphINVENT are $85.4 \%$ 228 and $95.3 \%$ respectively, which are lowest among all the models. In order to check how much 229 duplication is generated among the sample sets, the repetition rate $\left(R_{\text {rept }}\right)$ was calculated via the 230 formula below:

$$
R_{\text {rept }}=\frac{N_{\text {valid }}-N_{\text {unique }}}{N_{\text {unique }}},
$$

232

where, $N_{\text {valid }}$ is the number of total valid molecules in the $1 \mathrm{~B}$ generated set and $N_{\text {unique }}$ is the number of unique valid molecules in the $1 \mathrm{~B}$ generated set (i.e. duplicates removed). The compound repetition rates of most deep generative models were around 1.0, that is to say, most compounds were sampled twice on the average. ORGAN and CharRNN have the highest repetition rates, which are 3.8 and 1.4 respectively, whereas GraphINVENT and LatentGAN have the lowest (0.7). It seems that all the deep generative models had a satisfactory high percent validity that was above $85 \%$ in this study. The validity of CharRNN reached as high as $99.7 \%$. ORGAN had a repetitive rate as high as 3.8, which means that each generated compound was sampled 4.8 times on average. The high repetition rate resulted in a low overall compound coverage for ORGAN, where the coverage was as low as $16 \%$.

Table 2. Percentage of the valid molecules and molecular repetition rate in the 1B generated set for each model in this study. The uncertainty in the percent validity was less than a fraction of a percentage point for each model. 


\begin{tabular}{llllllll}
\hline Model & REINVENT & CharRNN & AAE & ORGAN & LatentGAN & VAE & GraphINVENT \\
\hline $\begin{array}{l}\text { Validity } \\
(\%)\end{array}$ & 99.3 & 99.7 & 97.8 & 97.2 & 85.4 & 98.2 & 95.3 \\
$\begin{array}{l}\text { Repetition } \\
\text { rate }\end{array}$ & 0.9 & 1.4 & 0.9 & 3.8 & 0.7 & 1.0 & 0.7 \\
\hline
\end{tabular}

\section{Coverage of GDB-13 chemical space}

247 The molecule and substructure coverage of GDB-13 space for all generative models studied herein 248 is shown in Figure 4a. It can be seen that all the models possess good capabilities for generalization, 249 surpassing the coverage of the $1 \mathrm{M}$ training set used, which has a $\sim 0.1 \%$ coverage of GDB-13 250 compounds, $\sim 0.9 \%$ coverage of GDB-13 ring systems, and $\sim 0.9 \%$ coverage of GDB-13 functional 251 groups. REINVENT achieves the highest compound and FG coverage (39\% and 26\%, 252 respectively), while AAE achieves best RS coverage (41\%). The GAN models (ORGAN and 253 LatentGAN) have lowest coverage at all three levels.

254 Using these new metrics, the difference in performance among these models is more pronounced; 255 this is in contrast to a previous benchmarking study using the MOSES metrics, ${ }^{[10]}$ where the two 256 GAN models appear to perform similarly with the CharRNN, AAE, and VAE models.

257 Overall, REINVENT, CharRNN, AAE, and VAE are the top-ranking models in this benchmarking 258 study. They have a compound coverage, RS coverage, and FG coverage around 34\%, 34\%, and $25921 \%$, respectively, in all cases. The performance of GraphINVENT is in the middle rank among 260 the generative models in this study, and demonstrates coverage scores of $22 \%, 30 \%$, and $24 \%$ for 261 compound coverage, RS coverage, and FG coverage, respectively. 
(a)

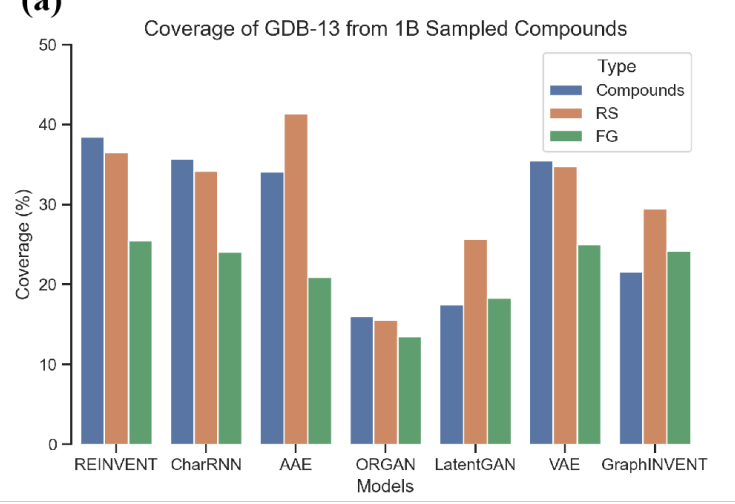

(b)

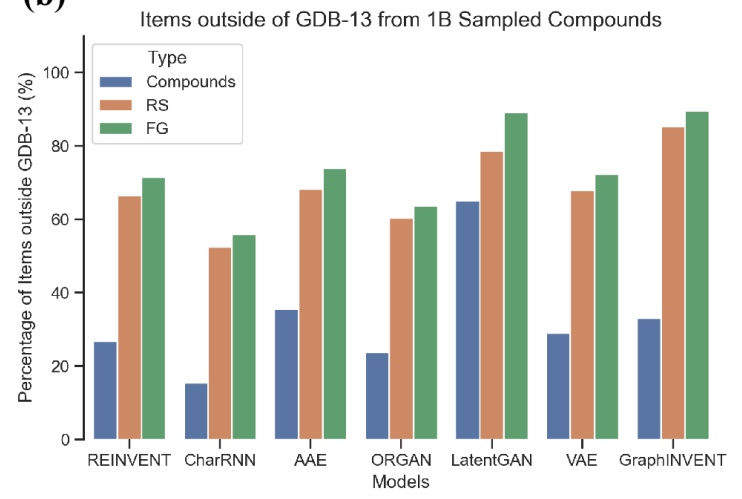

267 Figure 4. Coverage of GDB-13 chemical space using 1B sampled molecules. (a) Coverage of 268 compounds, ring systems (RS), and functional groups (FG) in GDB-13 ( $\left.P_{\text {covered }}\right)$. (b) Percentage 269 of sampled molecules, RS, and FG that are outside the chemical space of GDB-13 $\left(P_{\text {out }}\right)$.

270 Coverage of compounds, RS, and FG in GDB-13 was calculated via the formula below:

$$
P_{\text {covered }}=\frac{N_{\text {unique_in }}}{N_{G D B 13}} * 100 \%,
$$

where $N_{\text {unique_in }}$ is the number of unique valid sampled compounds, RS, or FG that are also found

273 in GDB-13, and $N_{G D B 13}$ is the total number of compounds, RS, or FG present in GDB-13.

274 The percentage of sampled compounds, RS, or FG that are outside the chemical space of GDB-13

275 was calculated via the formula below:

$$
P_{\text {out }}=\frac{N_{\text {unique_out }}}{N_{\text {unique }}} * 100 \% \text {, }
$$

277 where $N_{\text {unique_out }}$ is the number of unique valid sampled compounds, RS, or FG that are not found 278 in GDB-13, and $N_{\text {unique }}$ is the total number of unique valid compounds, RS, or FG in the generated 279 sets. 
There are four major metrics mentioned above, namely validity, repetition rate, coverage of GDB13 chemical space, and percentage outside GDB-13. Validity represents how good a generative model has learned the chemical rules for constructing compounds; repetition rate represents how much structure duplication exists in the generated compound set; generalization capacities of models can be measured with the coverage of GDB-13 after being trained on a smaller fraction of chemical space. As a supplement to above metrics, percentage outside GDB-13 shows how many sampled compounds fall outside the scope of GDB-13 (which are usually non drug-like compounds). Also, these four metrics are not independent from each other. For example, if a model has a high validity and a small percentage sampled outside GDB-13, given that exactly 1B compounds are sampled, the only reasonable explanation for a low GDB-13 coverage is a high repetition rate.

Figure $4 \mathrm{~b}$ shows the generated structures outside GDB-13. As GDB-13 uses filters to remove molecules that do not satisfy simple chemical stability and synthetic feasibility rules, such as ringstrain criteria and valency rules, there are many structures that can be generated which violate the filters used by GDB-13. For example, there are around 27\% valid SMILES generated by REINVENT which fall outside the scope of GDB-13 chemical space. However, for CharRNN, only $15 \%$ of its respective generated sets fall outside GDB-13, which is lower than other models in this study. As the percent validity of the structures generated by both models is above $97 \%$, we conclude that the lower fraction of compounds outside GDB-13 is due to the high repetition rate of compounds for these models, as shown in Table 2. As for the percentage of RS and FG outside of the scope of GDB-13, more than $50 \%$ of all FG and RS found in the generated sets for each model are outside the GDB-13 chemical space. 
After training with a subset of the GDB-13 database $(0.1 \%)$, all the generative models showed promising performance in terms of compound coverage. Around 16\% compounds of GDB-13 were covered with 1B SMILES sampled by the LatentGAN, which is 160 times greater than the coverage of the training dataset itself. The model with the best performance in this study is REINVENT, which has an observed compound coverage as high as $39 \%$. Thus, we conclude that deep generative models in general have satisfactory learning and generalization capacities. In terms of overall GDB-13 compound coverage, the rank of performance in descending order is REINVENT $>$ CharRNN $>$ VAE $>$ AAE $>$ GraphINVENT $>$ LatentGAN $>$ ORGAN.

The GDB-13 coverage of RS and FG was generally less than the coverage of compounds, except in the cases of AAE, LatentGAN, and GraphINVENT. However, in these cases, greater than $60 \%$ RS and FG in the generated set were outside the scope GDB-13 chemical space, while less than $40 \%$ of generated molecules were outside GDB-13 (except LatentGAN). In terms of RS coverage of GDB-13, the rank of performance in descending order is AAE $>$ REINVENT $>$ VAE $>$ CharRNN $>$ GraphINVENT $>$ LatentGAN $>$ ORGAN. In terms of FG coverage of GDB-13, the rank of performance in descending order is REINVENT $>$ VAE $>$ GraphINVENT $>$ CharRNN $>$ AAE $>$ LatentGAN $>$ ORGAN. Examples of the most commonly observed groups in structures generated by the two best models in terms of functional groups and ring systems recovery, REINVENT and AAE, are shown in Figures $6 \& 8$. Examples of the most commonly observed groups that are outside of GDB-13 in structures and generated by LatentGAN, are shown in Figures $7 \& 9$.

It is worthwhile to mention that the original LatentGAN adopts a heteroencoder and decoder model (DDC) trained on ChEMBL dataset, the LatentGAN had a compounds coverage, RS coverage and FG coverage of GDB-13 as 13\%, $15 \%$ and 18\%, respectively. When the DDC model were trained 
on a $3 \mathrm{M}$ subset of GDB-13 instead, the compounds coverage, RS coverage and FG coverage of

327 decoder model trained on the $3 \mathrm{M}$ subset in this study.

\section{Relationship between the coverage of GDB-13 and occurrence frequency}

(a)

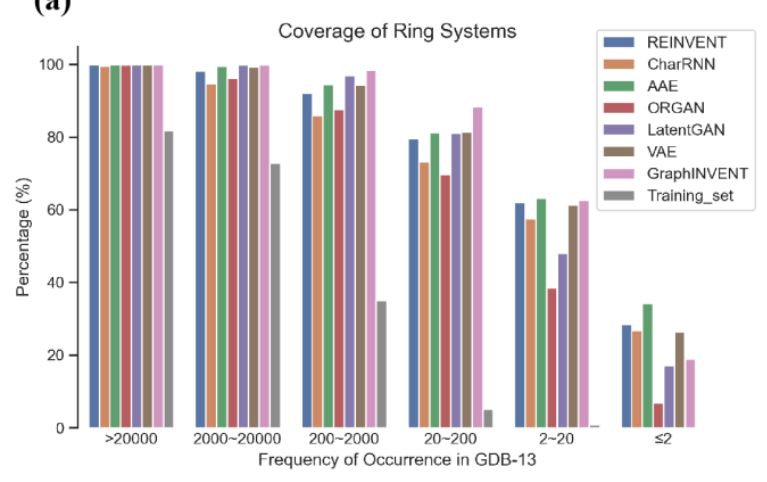

(c)

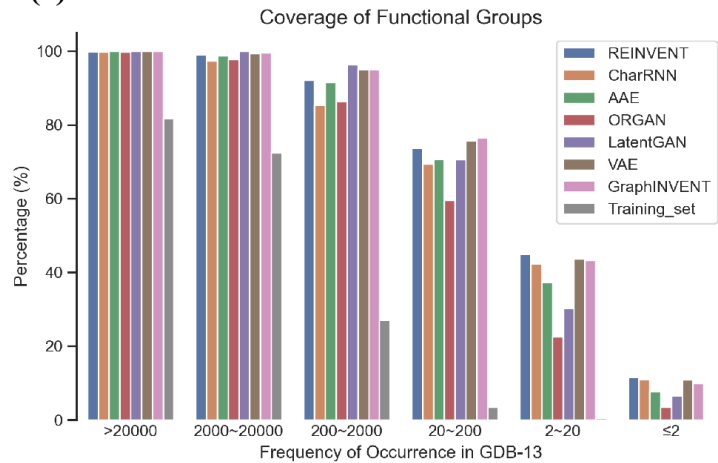

(b)

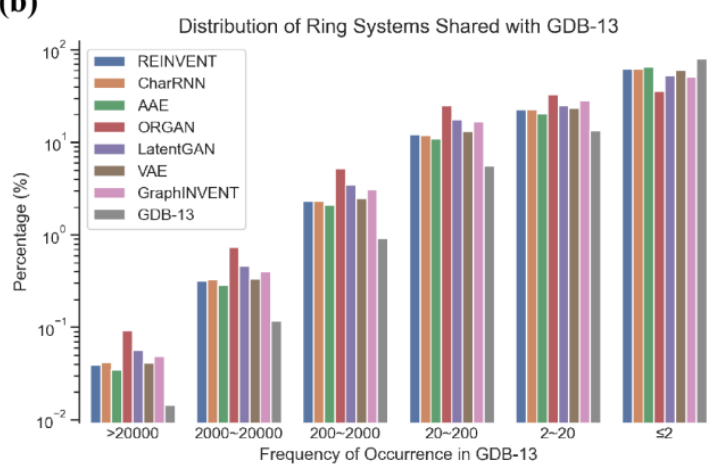

(d)

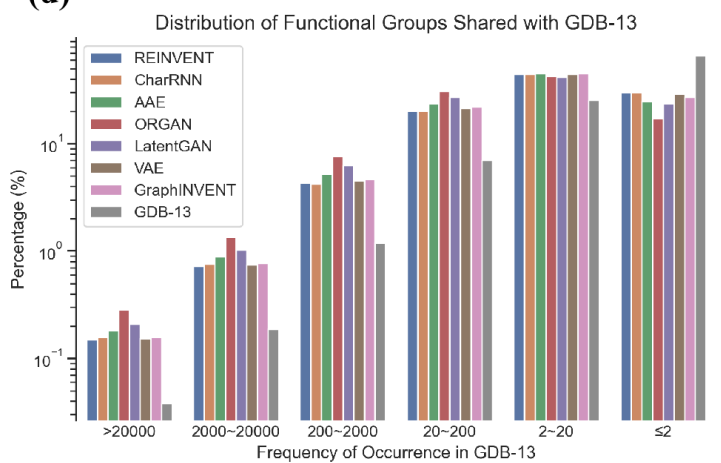

330

331 Figure 5. Coverage of GDB-13 chemical space from 1B sampled molecules, grouped by the 332 occurrence frequency of molecules in GDB-13. (a \& c) Coverage of RS and FG. (b \& d) 333 Distribution of generated RS and FG that are shared with the chemical space of GDB-13. The y334 axes for (b) and (d) are displayed in logarithmic scale.

335 The coverage of GDB-13 chemical space from 1B sampled molecules, grouped by the 336 occurrence frequency of molecules in GDB-13, was calculated via the formula below: 


$$
P_{\text {covered }}=\frac{N_{\text {unique_in }}\left(R_{m}, R_{n}\right)}{N_{G D B 13}\left(R_{m}, R_{n}\right)} * 100 \%,
$$

where $N_{\text {unique_in }}\left(R_{m}, R_{n}\right)$ is the number of unique RS or FG in the sampled set that have an occurrence frequency in the interval of $R_{m}-R_{n}$ (including $\left.R_{n}\right)$ in GDB-13, and $N_{G D B 13}\left(R_{m}, R_{n}\right)$ is the total number of RS or FG in GDB-13 with an occurrence frequency in the interval of $R_{m}-$ $R_{n}$ (including $R_{n}$ ). As such, $P_{\text {covered }}$ represents the coverage of specific set of substructures $N_{G D B 13}\left(R_{m}, R_{n}\right)$ of GDB-13 from the 1B generated set.

In Figures 5a and 5c, the RS and FG coverage of various models is broken down into different frequency sections to examine the coverage performance for different types of substructures. Figure 5 shows that for high frequency RS and FG, the coverage is high and quite similar among all models, while for less frequent RS and FG, the coverage reveals differences between models. On the other hand, comparing with the training set, all models demonstrate clear enrichment of RS and FG coverage, and the enrichment gets bigger as the RS and FG frequency is lower. As for RS and FG at the occurrence ranges of ">20000", "2000-20000", and "200-2000", the coverage is close to $100 \%$ for all models, while the coverage of the training dataset is around $82 \%, 73 \%$, and $31 \%$ at these respective occurrence frequency ranges. As for RS at the occurrence range of "20200", "2-20" and " $\leq 2$ ", most generative models have an RS coverage of around $80 \%, 60 \%$, and $30 \%$, compared to only $5 \%, 1 \%$, and $0 \%$ for the training dataset. The coverage of FG at the different occurrence frequency ranges has a similar pattern to the RS coverage.

Similarly, distribution of generated RS and FG that are shared with the chemical space of GDB13 was calculated via the formula below:

$$
P_{\text {dist }}=\frac{N_{\text {unique_in }}\left(R_{m}, R_{n}\right)}{N_{\text {unique_in }}} * 100 \%,
$$


358 where $N_{\text {unique_in }}\left(R_{m}, R_{n}\right)$ is the number of unique RS or FG in the sampled set that have an 359 occurrence frequency in the range of $R_{m}$ to $R_{n}$ in GDB-13, and $N_{\text {unique_in }}$ is the total number of 360 unique RS or FG in the generated set, which are also included in GDB-13. Thus, $P_{\text {dist }}$ is a metric 361 of the distribution of RS or FG that are shared with GDB-13 at different occurrence ranges.

362 The distributions of generated RS and FG corresponding to occurrence frequency in GDB-13 are 363 shown in Figures $5 \mathrm{~b} \& 5 \mathrm{~d}$. Given that most RS and FG have an occurrence frequency below 20 in 364 the GDB-13 database (as shown in Figure 1), the overall coverage of RS and FG is thus dominated 365 by ones with low occurrence frequency.

366 The most frequent and least frequent ring systems and functional groups sampled by the deep 367 generative models are listed in Figures 6-9. The most often sampled ring systems are simple carbon 368 cycles or aromatic heterocycles containing $\mathrm{O}$ and $\mathrm{N}$ atoms, such as $\mathrm{C} 1 \mathrm{CC} 1$ (cyclopropane), which 369 was sampled up to $78 \mathrm{M}$ times in the 1B sample set, and $\mathrm{C} 1 \mathrm{COC} 1$ (oxetane), which were sampled up to $26 \mathrm{M}$ times in the $1 \mathrm{~B}$ sample set. For comparison, the benzene ring ranked $85^{\mathrm{th}}$ among the most common sampled ring systems. As for the least common sampled ring systems, they were 372 usually complex macrocycles that were only sampled once out of the $1 \mathrm{~B}$ compounds generated.

373 The most commonly sampled functional groups are ordinary small ones, such as single oxygen and nitrogen atoms, $\mathrm{C}-\mathrm{C}$ double bonds, and $\mathrm{C}-\mathrm{C}$ triple bonds. The least commonly sampled 375 functional groups are those with complex structures formed by a combination of simple ones. The 376 ring systems and functional groups that are not included in GDB-13 usually do not conform to 377 simple chemical stability and synthetic feasibility rules.

378 Most of the RS ( $\sim 93 \%)$ and FG ( $91 \%)$ found in the generated sets that are also found in GDB-13 379 are seen less than 20 times. As the results show in Figure 2, RS and FG that occur more frequently 380 in GDB-13 tend to have smaller fragment weights. The building blocks of RS and FG are basic 
381 rings and functional groups with simple structures and small fragment weights. More complex RS

382 and FG can be built via the combination of these basic components.

383 The coverage of RS and FG with an occurrence frequency in GDB-13 greater than 200 was nearly

$384100 \%$. This is because these RS and FG can be easily obtained via combinations of smaller

385 fragments. However, given that as many as up to 13 heavy atoms were considered in constructing

386 the GDB-13 database, most RS and FG possess complex structures and were included in

387 compounds of GDB-13 less than 20 times. RS and FG that occur less than 20 times in the generated

388 sets dominate the coverage of the deep generative models.

389 Besides, as shown in Figures 10, most common ring systems and functional groups sampled by 390 generative models have close relative occurrence frequency compared to their distribution in 391 GDB-13.

\section{Model comparison}

393 It is interesting to observe that these models describe the chemical space so differently, although

394 trained with the same training set. It seems that the RS and FG coverage of GraphINVENT is 395 higher than its overall molecular coverage, one reason could be due to its large action space; that 396 is, the number of possible "correct" sampled actions at any stage during graph generation is much 397 larger than it is for SMILES-based methods which must use only tokens sampled in the training 398 set. As such, given that GraphINVENT samples actions probabilistically, it is possible that 399 sequences of actions are sampled which have never been seen in the training set, thus leading to 400 new molecules. Another interesting observation is that GAN based models generally perform 401 worst in terms of GDB-13 coverage on all three metrics, one reason could be due to that, in the 402 adversarial training, the generator is supposed to mimic the true data as much as possible to fool 403 the discriminator, which deteriorates its generalization capability to a certain extent. We also 

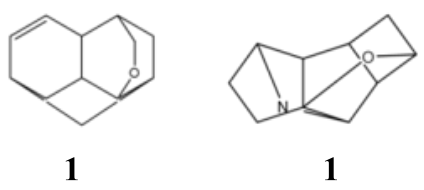

1

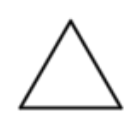

$78,827,335$

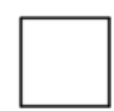

$30,215,635$ sampled compounds.

\section{Typical ring systems sampled that are included in GDB-13}

Most common sampled by AAE

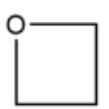

$26,482,475$

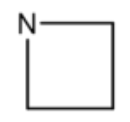

$23,670,090$

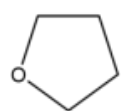

$17,939,880$

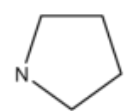

$14,351,285$

Least common sampled by AAE

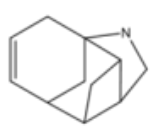

1

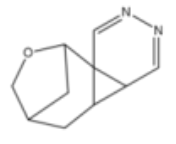

1

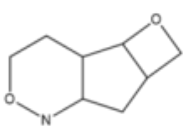

1

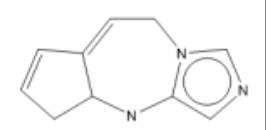

1

Figure 6. Typical ring systems that are sampled by AAE, which are included in GDB-13. The numbers below the structures in the figure are the occurrence frequency of ring systems in the $1 \mathrm{~B}$

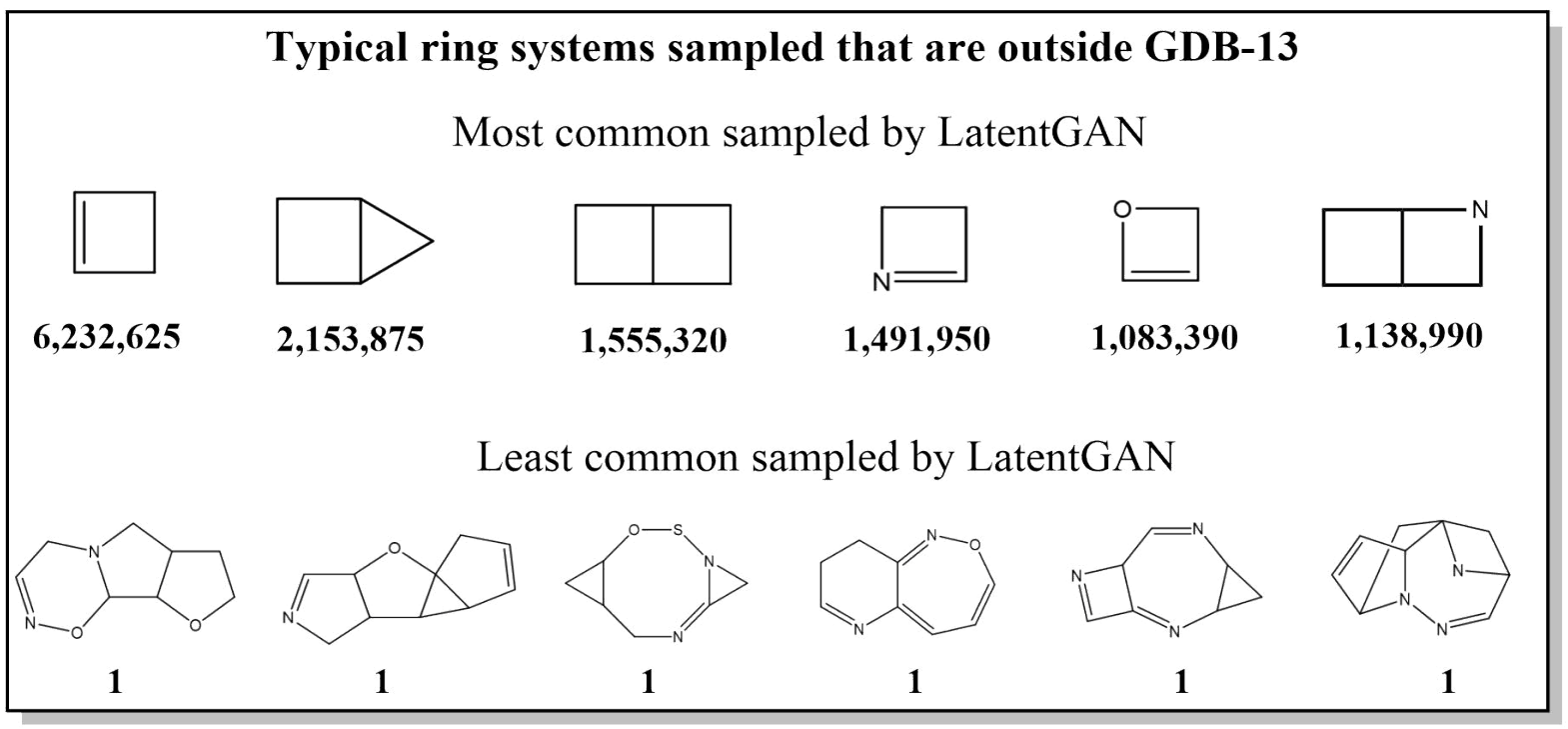

Figure 7. Typical ring systems that are sampled by LatentGAN, which are outside GDB-13. 


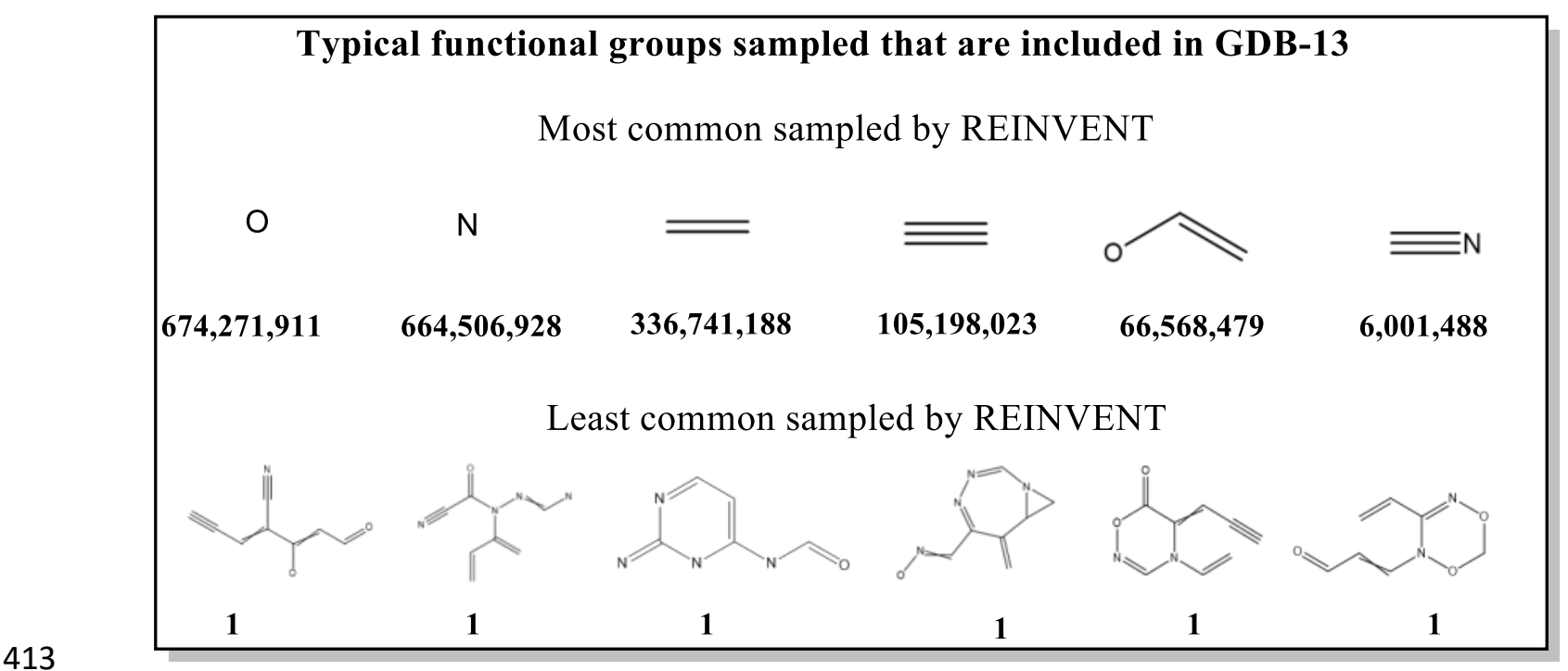

414 Figure 8. Typical functional groups that are sampled by REINVENT, which are included in GDB41513

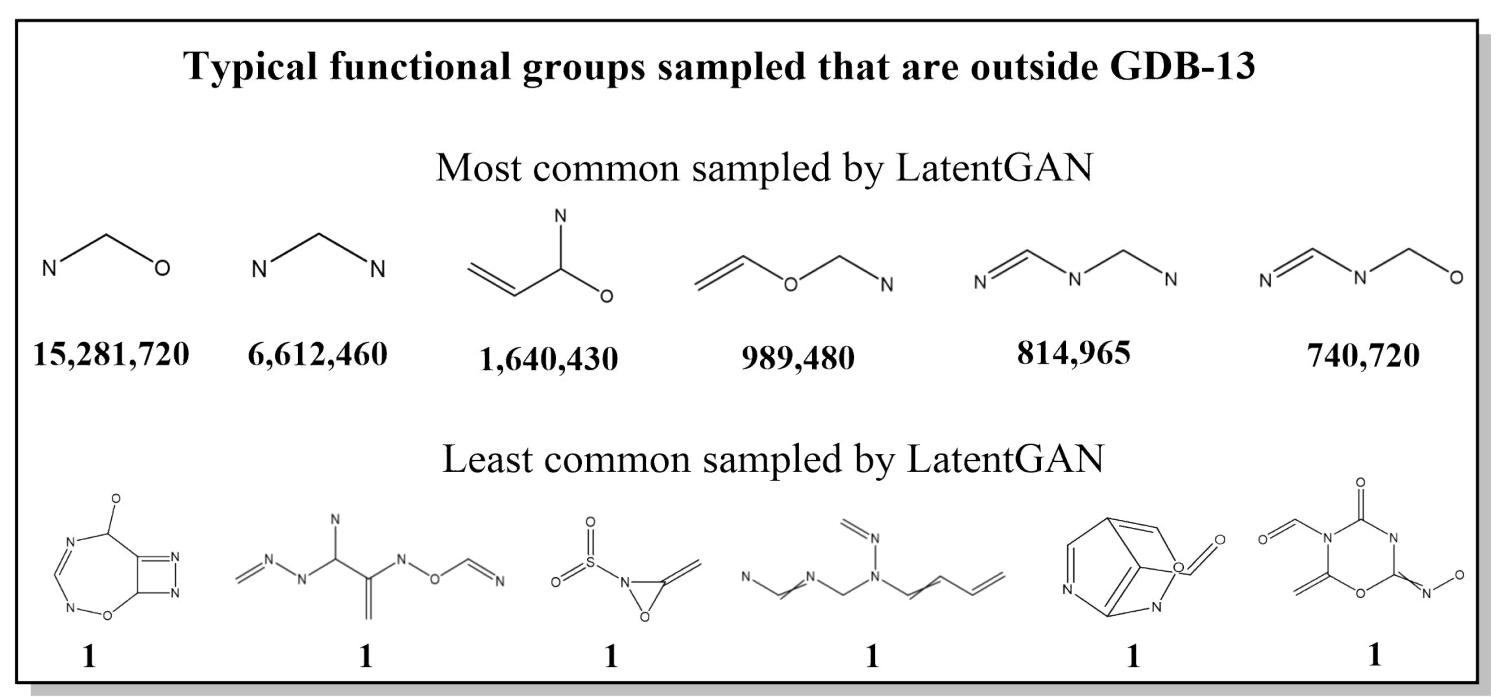

418 Figure 9. Typical functional groups that are sampled by LatentGAN, which are outside GDB-13. 

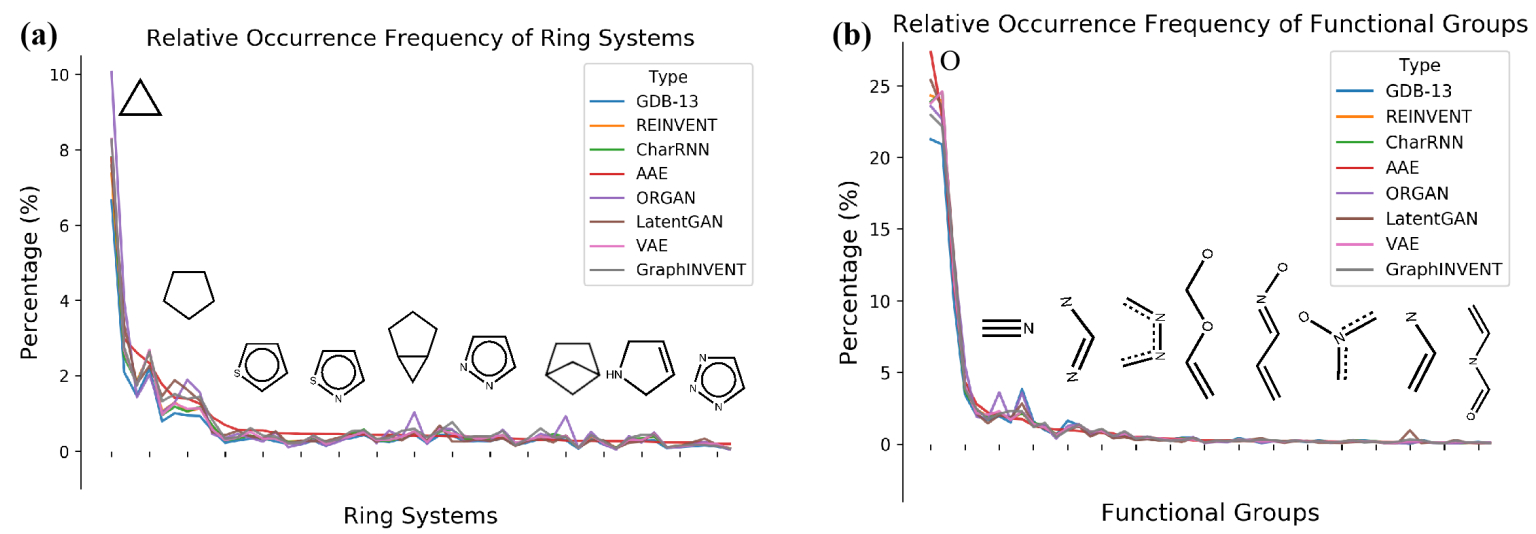

Figure 10. Relative occurrence frequency of most common functional groups and ring systems.

\section{Conclusions}

422 Molecules consist of a variety of ring systems and functional groups, which are connected in 423 different ways to form molecules. The most basic ring systems and functional groups have simple 424 structures and small fragment weights; these can be found in GDB-13 molecules over dozens of times. More complex ring systems and functional groups have complicated structures and large

426 fragment weights, and might only occur in GDB-13 a handful of times. However, due to their 427 structural variety and enormous quantity (>90\%), complex ring systems and functional groups are 428 strong components affecting the coverage of GDB-13.

429 All the deep generative models studied in this work have over 100 times greater chemical space 430 coverage for GDB-13 using 1B samples than the training set (1M) used to train the models. In 431 terms of compound coverage of GDB-13, the best model (REINVENT) reached $39 \%$ coverage, 432 far beyond the coverage of ORGAN $(\sim 16 \%)$, which ranked lowest amongst the models in this 433 study. Depending on the generative task, the deep generative model used should thus be chosen 
434 carefully, as there are differences in how all these seemingly similar models sample the chemical 435 space.

436 Associated Content

437 Author Information

438 Corresponding Author

439 Hongming Chen, E-mail: chen_hongming@grmh-gdl.cn

440 Author Contributions

441 J. Z. ran training and generation jobs using REINVENT, CharRNN, VAE; LatentGAN, and

442 ORGAN. R. M. ran training and generation jobs using GraphINVENT. R. M. and J. Z. ran

443 benchmarking calculations for this work, and J. Z. made all figures. The manuscript was written 444 through the contributions of all authors. All authors have given approval to the final version of the 445 manuscript.

446 Acknowledgements

447 J. Z. would like to acknowledge funding provided by XtalPi Inc, and R. M. thanks the Postdoc 448 Program at AstraZeneca.

449 Supplementary Materials

450 The detailed hyperparameters and training loss curves of all models can be found in supplementary 451 materials. The training, sampling, and analysis script could found in the GitHub repository, 452 https://github.com/jeah-z/Generative Models benchmark gdb13.

453 Abbreviations 
RS, Ring system(s); FG, Functional group(s); GAN, Generative adversarial network; GNN,

graph neural network; RNN, recurrent neural network.

\section{References}

(1) Ciregan, D.; Meier, U.; Schmidhuber, J. Multi-Column Deep Neural Networks for Image Classification. In 2012 IEEE conference on computer vision and pattern recognition, 2012; IEEE: 2012; pp 3642-3649.

(2) Krizhevsky, A.; Sutskever, I.; Hinton, G. E. Imagenet Classification with Deep Convolutional Neural Networks. In Advances in neural information processing systems, 2012; 2012; pp 1097-1105.

(3) Taigman, Y.; Yang, M.; Ranzato, M. A.; Wolf, L. Deepface: Closing the Gap to Human-Level Performance in Face Verification. In Proceedings of the IEEE conference on computer vision and pattern recognition, 2014; 2014; pp 1701-1708.

(4) Silver, D.; Huang, A.; Maddison, C. J.; Guez, A.; Sifre, L.; Van Den Driessche, G.; Schrittwieser, J.; Antonoglou, I.; Panneershelvam, V.; Lanctot, M., Mastering the Game of Go with Deep Neural Networks and Tree Search. nature 2016, 529, 484-489.

(5) Hadjeres, G.; Pachet, F.; Nielsen, F. Deepbach: A Steerable Model for Bach Chorales Generation. In International Conference on Machine Learning, 2017; PMLR: 2017; pp 1362-1371.

(6) Garg, S.; Rish, I.; Cecchi, G.; Lozano, A., Neurogenesis-Inspired Dictionary Learning: Online Model Adaption in a Changing World. arXiv preprint arXiv:1701.06106 2017.

(7) Johnson, M.; Schuster, M.; Le, Q. V.; Krikun, M.; Wu, Y.; Chen, Z.; Thorat, N.; Viégas, F.; Wattenberg, M.; Corrado, G., Google's Multilingual Neural Machine Translation System: Enabling Zero-Shot Translation. Transactions of the Association for Computational Linguistics 2017, 5, 339-351.

(8) Bjerrum, E. J.; Threlfall, R., Molecular Generation with Recurrent Neural Networks (Rnns). arXiv preprint arXiv:1705.04612 2017.

(9) Kotsias, P.-C.; Arús-Pous, J.; Chen, H.; Engkvist, O.; Tyrchan, C.; Bjerrum, E. J., Direct Steering of De Novo Molecular Generation with Descriptor Conditional Recurrent Neural Networks. Nature Machine Intelligence 2020, 2, 254-265.

(10) Polykovskiy, D.; Zhebrak, A.; Sanchez-Lengeling, B.; Golovanov, S.; Tatanov, O.; Belyaev, S.; Kurbanov, R.; Artamonov, A.; Aladinskiy, V.; Veselov, M.; Kadurin, A.; Johansson, S.; Chen, H.; Nikolenko, S.; AspuruGuzik, A.; Zhavoronkov, A., Molecular Sets (Moses): A Benchmarking Platform for Molecular Generation Models. Front Pharmacol 2020, 11, 565644.

(11) Prykhodko, O.; Johansson, S. V.; Kotsias, P.-C.; Arús-Pous, J.; Bjerrum, E. J.; Engkvist, O.; Chen, H., A De Novo Molecular Generation Method Using Latent Vector Based Generative Adversarial Network. Journal of Cheminformatics 2019, 11, 74.

(12) Schneider, G.; Fechner, U., Computer-Based De Novo Design of Drug-Like Molecules. Nat Rev Drug Discov 2005, 4, 649-63.

(13) DiMasi, J. A.; Grabowski, H. G.; Hansen, R. W., Innovation in the Pharmaceutical Industry: New Estimates of R\&D Costs. Journal of health economics 2016, 47, 20-33.

(14) Patel, H.; Bodkin, M. J.; Chen, B.; Gillet, V. J., Knowledge-Based Approach to De Novo Design Using Reaction Vectors. Journal of chemical information and modeling 2009, 49, 1163-1184.

(15) Schneider, G.; Lee, M.-L.; Stahl, M.; Schneider, P., De Novo Design of Molecular Architectures by Evolutionary Assembly of Drug-Derived Building Blocks. Journal of computer-aided molecular design 2000, 14, 487-494.

(16) Spiegel, J. O.; Durrant, J. D., Autogrow4: An Open-Source Genetic Algorithm for De Novo Drug Design and Lead Optimization. Journal of Cheminformatics 2020, 12, 1-16. 
(17) Chen, H.; Engkvist, O.; Wang, Y.; Olivecrona, M.; Blaschke, T., The Rise of Deep Learning in Drug 498 Discovery. Drug discovery today 2018, 23, 1241-1250.

(18) Blaschke, T.; Arus-Pous, J.; Chen, H.; Margreitter, C.; Tyrchan, C.; Engkvist, O.; Papadopoulos, K.; Patronov, A., Reinvent 2.0: An Ai Tool for De Novo Drug Design. J Chem Inf Model 2020, 60, 5918-5922.

(19) Blaschke, T.; Olivecrona, M.; Engkvist, O.; Bajorath, J.; Chen, H., Application of Generative Autoencoder in De Novo Molecular Design. Molecular informatics 2018, 37, 1700123.

502

(20) Guimaraes, G. L.; Sanchez-Lengeling, B.; Outeiral, C.; Farias, P. L. C.; Aspuru-Guzik, A., ObjectiveReinforced Generative Adversarial Networks (Organ) for Sequence Generation Models. arXiv preprint arXiv:1705.10843 2017.

(21) Kadurin, A.; Aliper, A.; Kazennov, A.; Mamoshina, P.; Vanhaelen, Q.; Khrabrov, K.; Zhavoronkov, A., The Cornucopia of Meaningful Leads: Applying Deep Adversarial Autoencoders for New Molecule Development in Oncology. Oncotarget 2017, 8, 10883.

509 (22) Grisoni, F.; Moret, M.; Lingwood, R.; Schneider, G., Bidirectional Molecule Generation with Recurrent Neural Networks. Journal of chemical information and modeling 2020, 60, 1175-1183.

(23) Gupta, A.; Müller, A. T.; Huisman, B. J.; Fuchs, J. A.; Schneider, P.; Schneider, G., Generative Recurrent Networks for De Novo Drug Design. Molecular informatics 2018, 37, 1700111. Reinforcement Learning. J Cheminform 2017, 9, 48.

(25) Segler, M. H.; Kogej, T.; Tyrchan, C.; Waller, M. P., Generating Focused Molecule Libraries for Drug Discovery with Recurrent Neural Networks. ACS central science 2018, 4, 120-131.

(26) Yuan, Q.; Santana-Bonilla, A.; Zwijnenburg, M. A.; Jelfs, K. E., Molecular Generation Targeting Desired Electronic Properties Via Deep Generative Models. Nanoscale 2020, 12, 6744-6758.

(27) Kalchbrenner, N.; Grefenstette, E.; Blunsom, P., A Convolutional Neural Network for Modelling Sentences. arXiv preprint arXiv:1404.2188 2014.

(28) Kingma, D. P.; Welling, M., Auto-Encoding Variational Bayes. arXiv preprint arXiv:1312.6114 2013.

(29) Kusner, M. J.; Paige, B.; Hernández-Lobato, J. M., Grammar Variational Autoencoder. arXiv preprint arXiv:1703.01925 2017.

(30) Brown, N.; Fiscato, M.; Segler, M. H.; Vaucher, A. C., Guacamol: Benchmarking Models for De Novo Molecular Design. Journal of chemical information and modeling 2019, 59, 1096-1108.

(31) Blum, L. C.; Reymond, J.-L., 970 Million Druglike Small Molecules for Virtual Screening in the Chemical Universe Database Gdb-13. Journal of the American Chemical Society 2009, 131, 8732-8733.

(32) Arus-Pous, J.; Blaschke, T.; Ulander, S.; Reymond, J. L.; Chen, H.; Engkvist, O., Exploring the Gdb-13 Chemical Space Using Deep Generative Models. J Cheminform 2019, 11, 20.

(33) Ertl, P.; Altmann, E.; McKenna, J. M., The Most Common Functional Groups in Bioactive Molecules and How Their Popularity Has Evolved over Time. Journal of Medicinal Chemistry 2020, 63, 8408-8418.

(34) Taylor, R. D.; MacCoss, M.; Lawson, A. D., Rings in Drugs. J Med Chem 2014, 57, 5845-59.

(35) Arús-Pous, J.; Johansson, S. V.; Prykhodko, O.; Bjerrum, E. J.; Tyrchan, C.; Reymond, J.-L.; Chen, H.; Engkvist, O., Randomized Smiles Strings Improve the Quality of Molecular Generative Models. Journal of Cheminformatics 2019, 11.

(36) Gómez-Bombarelli, R.; Wei, J. N.; Duvenaud, D.; Hernández-Lobato, J. M.; Sánchez-Lengeling, B.; Sheberla, D.; Aguilera-Iparraguirre, J.; Hirzel, T. D.; Adams, R. P.; Aspuru-Guzik, A., Automatic Chemical Design Using a Data-Driven Continuous Representation of Molecules. ACS central science 2018, 4, 268276.

540 (37) Makhzani, A.; Shlens, J.; Jaitly, N.; Goodfellow, I.; Frey, B., Adversarial Autoencoders. arXiv preprint 541 arXiv:1511.05644 2015.

542 (38) Mercado, R.; Rastemo, T.; Lindelöf, E.; Klambauer, G.; Engkvist, O.; Chen, H.; Bjerrum, E. J., Practical 543 Notes on Building Molecular Graph Generative Models. ChemRxiv 2020, Preprint.

544 (39) Landrum, G., Rdkit: Open-Source Cheminformatics. 2006. 
545 (40) Ertl, P., An Algorithm to Identify Functional Groups in Organic Molecules. Journal of cheminformatics 546 2017, 9, 1-7.

547 (41) Arús-Pous, J. Reinvent-Randomized. https://github.com/undeadpixel/reinvent-randomized 548 (accessed Sep 1, 2020).

549 (42) Polykovskiy, D.; Zhebrak, A.; Sanchez-Lengeling, B.; Golovanov, S.; Tatanov, O.; Belyaev, S.; Kurbanov, 550 R.; Artamonov, A.; Aladinskiy, V.; Veselov, M. Moses. https://github.com/molecularsets/moses (accessed 551 May 20, 2020).

552 (43) Johansson, S.; Prykhodko, O. Latent-Gan. https://github.com/Dierme/latent-gan (accessed Jan 5, 553 2021).

554 (44) Kotsias, P.; Bjerrum, E. J. Deepdrugcoder. https://github.com/pcko1/Deep-Drug-Coder (accessed Jan 555 5, 2021).

556 (45) Mercado, R.; Rastemo, T.; Lindelöf, E. Graphinvent. https://github.com/MolecularAl/GraphINVENT/ 557 (accessed Oct 20, 2020).

558 (46) Rocío, M.; Tobias, R.; Edvard, L.; Günter, K.; Ola, E.; Hongming, C.; Esben Jannik, B., Graph Networks 559 for Molecular Design. ChemRxiv 2020, Preprint.

560 (47) Oliphant, T. E., Python for Scientific Computing. Computing in Science \& Engineering 2007, 9, 10-20.

561 (48) Paszke, A.; Gross, S.; Massa, F.; Lerer, A.; Bradbury, J.; Chanan, G.; Killeen, T.; Lin, Z.; Gimelshein, N.; 562 Antiga, L. Pytorch: An Imperative Style, High-Performance Deep Learning Library. In Advances in neural 563 information processing systems, 2019; 2019; pp 8026-8037.

564 (49) Panaretos, V. M.; Zemel, Y., Statistical Aspects of Wasserstein Distances. Annual review of statistics 565 and its application 2019, 6, 405-431.

566 (50) Virtanen, P.; Gommers, R.; Oliphant, T. E.; Haberland, M.; Reddy, T.; Cournapeau, D.; Burovski, E.; 567 Peterson, P.; Weckesser, W.; Bright, J., Scipy 1.0: Fundamental Algorithms for Scientific Computing in 568 Python. Nature methods 2020, 17, 261-272.

569

570 


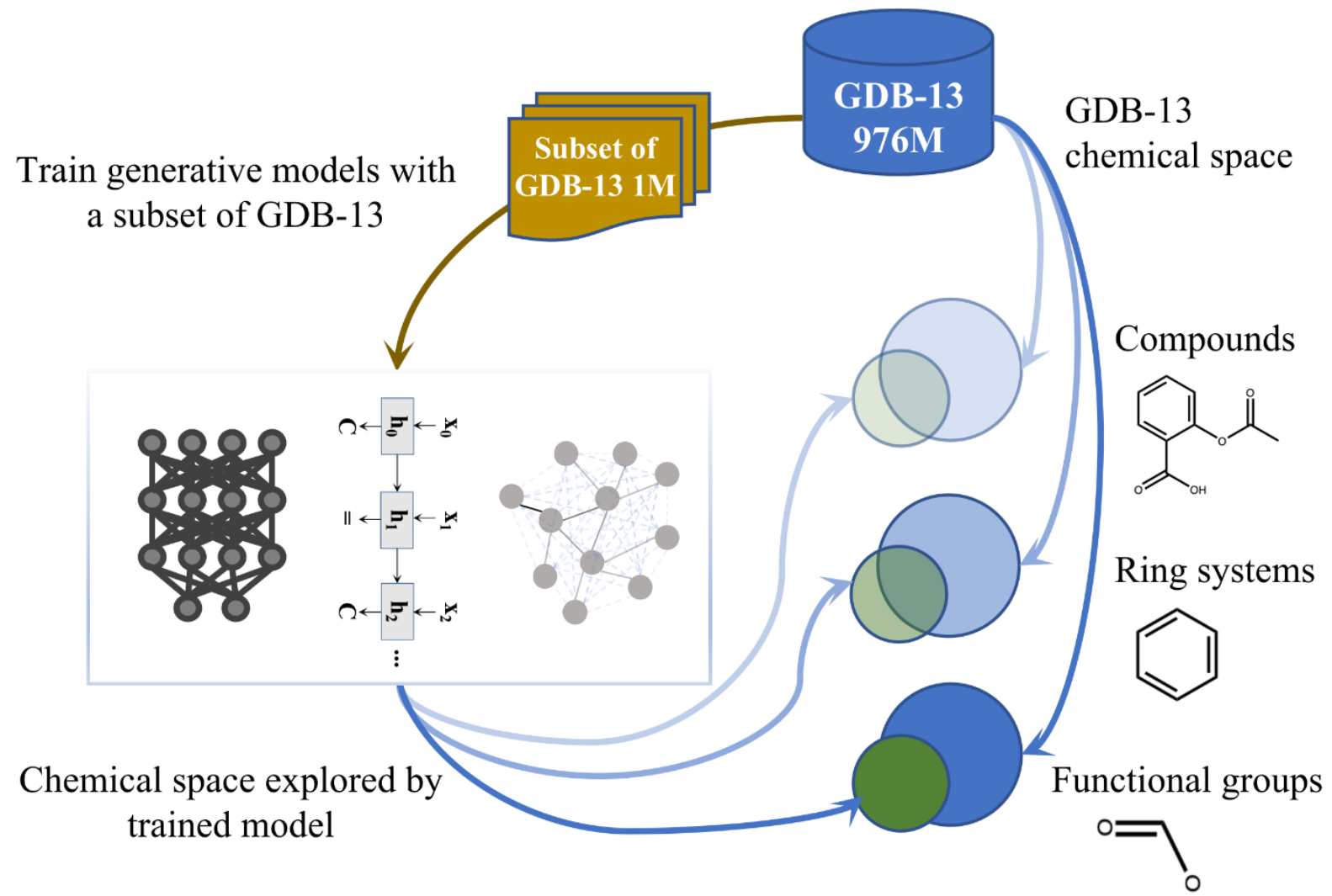

